\title{
Caracterización de los casos de ACV hemorrágico en la población pediátrica mayor de 1 mes en un hospital pediátrico de alta complejidad en la ciudad de Bogotá, Colombia durante los años 2012 a 2017
}

\author{
Characterization of hemorrhagic stroke in the pediatric population \\ greater than 1 month of age in a high complexity hospital in Bogotá \\ City, Colombia, from 2012 to 2017
}

\author{
Diego Muñoz-Suárez MD. ${ }^{1}$, Santiago Moreno-García MD. ${ }^{2}$, Orlando Faber Peláez MD. ${ }^{3}$, \\ Jaime Arias-Guatibonza MD. ${ }^{4}$, Carlos Martínez-López MD. ${ }^{5}$, Víctor Hugo Bastos MD. ${ }^{6}$
}

\footnotetext{
${ }^{1}$ Neurocirujano, Universidad Nacional de Colombia. Bogotá, Colombia.

${ }^{2}$ Médico Cirujano, Médico Hospitalario de Neurocirugía. Hospital Occidente de Kennedy. Universidad Nacional de Colombia. Bogotá, Colombia.

${ }^{3}$ Neurocirujano Pediátrico. Fundación Hospital La Misericordia. Bogotá, Colombia.

${ }^{4}$ Neurocirujano, Profesor titular. Universidad Nacional de Colombia. Bogotá, Colombia.

${ }^{5}$ Neurocirujano Pediátrico, Profesor titular. Universidad Nacional de Colombia. Bogotá, Colombia.

${ }^{6}$ Neurocirujano Endovascular, Coordinador del programa de especialización en Neurocirugía. Universidad Nacional de Colombia. Bogotá, Colombia.
}

\section{Resumen}

Introducción: Luego de revisar la literatura clínica disponible, no hay datos que indiquen la proporción de ACV infantil respecto a la población general, generando vacíos de conocimiento acerca del impacto de esta condición. Adicionalmente, teniendo en cuenta que más del $40 \%$ de los pacientes que sufren esta patología presentan un grado importante de discapacidad y una cuarta parta fallece secundariamente también. Asimismo, es una patología de gran importancia, pues hace parte de las diez principales causas de muerte en los niños. Objetivos: Describir las características demográficas clínicas, radiológicas, de tratamiento y de resultado de los casos de Ataque Cerebrovascular (ACV) de tipo hemorrágico en población pediátrica, en un hospital pediátrico de alta complejidad y centro de referencia a nivel nacional, entre los años 2012 y 2017 en la ciudad de Bogotá, Colombia. Métodos: Se revisarán de forma retrospectiva las historias clínicas de los pacientes que hayan ingresado por ACV hemorrágico en niños mayores de 1 mes y menores de 18 años durante el 2012 a $2017-I$ basados en un instrumento específico que permita recolectar las variables a estudio. Se realizó el análisis de las variables incluidas en el estudio obteniendo las frecuencias relativas de las características demográficas, clínicas, epidemiológicas y de resultado. Además, se realizó un análisis descriptivo de acuerdo a la naturaleza de la variable por medio de la herramienta Epilnfo 7 (CDC, 2008) y SPSS Statistics 22 para Windows. Resultados: Se revisaron 283 historias clínicas, de las cuales, después de aplicar los criterios de exclusión y revisión, se obtuvieron 55 pacientes como población del estudio. La mayor parte de los pacientes fueron adolescentes entre 9 y 14 años de edad. El hematoma intraparenquimatoso fue el hallazgo tomográfico más frecuentemente encontrado y las causas hematológicas como la leucemia, la hemofilia y otros trastornos hemorrágicos y de coagulación ocuparon cerca del $40 \%$ de la etiología de los eventos y en $25 \%$ de los casos no fue posible diagnosticar un proceso etiológico relacionado. En menos de la mitad de los casos, el manejo de la patología fue de carácter quirúrgico. La mortalidad de los pacientes fue del $38 \%$ de los casos y la discapacidad severa fue de alrededor del $12,8 \%$, medida con la escala de Rankin de 3 a 5. Puede existir alguna relación estadísticamente significativa entre el estado neurológico, el volumen del hematoma y la escala modificada de Rankin. Conclusiones: Se corroboró el aumento del riesgo de ACV

\section{Correspondencia a:}

Dr. Diego Muñoz, MD.

Dirección: Calle 39B Sur \# 72J - 45

diemu88@gmail.com 
hemorrágico en población pediátrica en pacientes con neoplasias y discrasias sanguíneas; además, del gran impacto para la salud pública y los costos económicos, debido a su gran morbilidad y discapacidad. Este es uno de los primeros estudios descriptivos para esta enfermedad subestimada. Colombia no cuenta con estadísticas claras de este problema, por lo que se requieren más estudios que permitan ampliar las correlaciones y pronósticos de esta patología.

Palabras clave: Stroke, hemorragia intracerebral, infantil, retrospectivo.

\section{Abstract}

Introduction: After reviewing the clinical literature, there are no data that indicate the proportion of childhood stroke compared to the general population, generating gaps in knowledge and the impact of this condition. Additionally, more than $40 \%$ of patients suffering from this pathology present an important degree of disability and a fourth part of them also die secondarily. Moreover, it is a pathology of great importance, since it is part of the ten main causes of death in children. Objectives: To describe the clinical, radiological, treatment and outcomes of hemorrhagic stroke in the pediatric population in a high complexity pediatric hospital between 2012 and 2017-I. Methods: During 2012 to 2017-I we retrospectively reviewed the clinical histories of children, older than 1 month and under 18 years, who were admitted at the hospital due to hemorrhagic stroke, based on a specific instrument that allows the variables to be collected. The variables included in the study were analyzed, obtaining the relative frequencies of the demographic, clinical, epidemiological characteristics and outcomes. Results: We reviewed 283 clinical histories and after applying the exclusion and revision criteria, 55 patients were obtained as study population. Most of the patients were teenager between 9 and 14 years of age. Intraparenchymal hematoma was the most frequent tomographic finding and hematologic causes such as leukemia, hemophilia and other bleeding and coagulation disorders occupied about $40 \%$ of the etiology of the events and in $25 \%$ of cases it was not possible to diagnose a related etiology. In less than half of the cases, the management of the pathology was surgical. The mortality of the patients was $38 \%$ of the cases and the severe disability was around $12.8 \%$, measured with the Rankin scale of 3 to 5 . There may be some statistically significant relationship between the neurological status, the volume of the hematoma and the modified Rankin scale. Conclusions: The increased risk of hemorrhagic stroke in the pediatric population was confirmed in patients with malignancies and blood dyscrasias; also, its great impact on public health and economic costs due to its great morbidity and disability. This is one of the first descriptive studies for this underestimated disease. Colombia does not have clear statistics on this problem, so more studies are required to expand the correlations and prognoses of this pathology.

Key words: Stroke, intracerebral hemorrhage, childhood, retrospective.

\section{Introducción}

El ataque cerebrovascular (ACV) tiene características clínicas y epidemiológicas distintas si se compara con los adultos. Es una patología de gran importancia pues hace parte de las diez principales causas de muerte en los niños ${ }^{1,2}$, siendo más frecuente en menores de 1 año debido principalmente a alteraciones pre y perinatales ${ }^{3,4}$. En países desarrollados es la tercera causa de muerte luego de las cardiopatías y los distintos tipos de cáncer ${ }^{5,6}$. Cada año se diagnostican aproximadamente 500.000 casos nuevos, con una incidencia que va desde 2,7 hasta $3,3 / 100.000$ habitantes $^{1}$. Dentro de las posibles causas de ACV en niños se encuentran las malformaciones vasculares (MV), enfermedades congénitas, hematopatías (anemia de células falciformes, trastornos de la coagulación), infecciosas (cada vez con menor incidencia) y en hasta el 30 al $50 \%$ de los casos no se encuentra una relación causa-efecto clara ${ }^{2,5,7-9}$. El diagnóstico se basa en la sospecha clínica, las condiciones genético-hereditarias de cada individuo y en la confirmación por medio de neuroimágenes. En cuanto al tratamiento, no se tienen en estos momentos protocolos establecidos para la población pediátrica y muchas de las alternativas actuales son extrapoladas de los conocimientos que se tienen en la población adulta ${ }^{10,11}$.
En nuestro medio no hay datos que indiquen la proporción de ACV infantil respecto a la población general, generando vacíos de conocimiento acerca del impacto de esta condición. Adicionalmente, teniendo en cuenta que más del $40 \%$ de los pacientes que sufren esta patología presentan un grado importante de discapacidad y una cuarta parta fallece secundariamente también ${ }^{1}$.

Se hace necesario realizar una caracterización de dichos pacientes, en un centro de referencia de impacto nacional. Esto con el fin de conocer las variables demográficas y posibles factores etiológicos relacionados, además de evaluar el tipo de manejo médico brindado y el enfoque terapéutico de una entidad en la que no se disponen guías internacionales de manejo aún.

\section{Objetivos}

Describir las características demográficas clínicas, radiológicas, de tratamiento y de resultado de los casos de Ataque Cerebrovascular (ACV) de tipo hemorrágico en población pediátrica en un hospital pediátrico de alta complejidad, centro de referencia a nivel nacional, en la ciudad de Bogotá, Colombia, entre los años 2012 y 2017-I. 


\section{Materiales y Métodos}

La naturaleza del estudio es retrospectiva y está dirigido a la población pediátrica mayor o igual a 1 mes, hasta los 18 años, que ingresen para estudio de ACV hemorrágico. No se consideraron pacientes menores de 1 mes, debido a que esta población hace parte del stroke perinatal, el cual, tiene un comportamiento, factores etiológicos (prematuridad principalmente) y enfoque clínico diferente. Por otra parte, el límite para la atención en pediatría es muy variable, dependiendo del lugar, el país, etc. Para tal efecto, por tratarse de un hospital, centro de referencia de todo el país para la población pediátrica, se utilizó el límite legal entre la dicha población (niños y adolescentes) y los adultos (mayores de 18 años). Teniendo en cuenta lo anterior, se revisarán las historias clínicas de los pacientes que ingresen al hospital y que cumplan los criterios establecidos.

\section{- Criterios de inclusión}

- Pacientes con ACV hemorrágico tratados en un hospital pediátrico de alta complejidad, centro de referencia a nivel nacional, en la ciudad de Bogotá, Colombia.

- Edad entre 1 mes y < 18 años.

- Que haya recibido el tratamiento completo en el hospital.

\section{- Criterios de exclusión}

- Pacientes mayores de 18 años.

- Pacientes con stroke perinatal (<1 mes).

- Pacientes que no hayan tenido tratamiento completo en el hospital.

- Pacientes del que no se disponga de la totalidad de la historia clínica.

Se realizara el análisis de las variables incluidas en el estudio obteniendo las frecuencias relativas de las características demográficas, clínicas, epidemiológicas y de resultado. Se realizara un análisis descriptivo de acuerdo a la naturaleza de la variable.

\section{Resultados}

\section{Características generales de la población a estudio}

Se revisaron 283 historias clínicas teniendo en cuenta los diagnósticos CIE-10 relacionados. Se excluyeron 222 pacientes debido a que no cumplían criterios y algunos de ellos no completaron el tratamiento en la institución. En una segunda revisión de las historias clínicas, se excluyeron 6 pacientes más, debido a que las historias se encontraban duplicadas por reingresos al hospital. En total, se obtuvieron 55 pacientes como población a estudio.

Los casos se presentaron de forma similar durante los años del estudio, con rango entre los 6 y 11 casos por año.

En cuanto al género de los pacientes estudiados, se distribuyeron en 33 pacientes masculinos y 22 pacientes femeninos. Los rangos de edad fueron variables, sin seguir una distribución normal ( $\mathrm{K}-\mathrm{S} \mathrm{p}=0,006)$ siendo aquellos niños entre los 9 y 14 años los que presentaron la patología con más frecuencia.

Teniendo en cuenta la mayor presentación de los eventos en la población adolescente, esto concuerda con el hallazgo de la escolaridad, siendo más frecuente los estudiantes de bachillerato $(52,7 \%)$ (Tabla 1$)$.

\section{Ingreso a urgencias}

Más del $45 \%$ de los pacientes que presentaron el evento hemorrágico, debutaron con Glasgow de 15, contrastado con cerca del $11 \%$ de estos que presentó Glasgow 3.

Los síntomas que caracterizaron los eventos fueron la cefalea con/sin emesis en $36,4 \%$ de los casos y el deterioro de la consciencia en el 38,2\% de los mismos. En el examen físico, la anisocoria se presentó en $13 \%$ de los casos y la sospecha de discrasias sanguíneas, ya sea por hallazgos semiológicos o pruebas de laboratorio de rutina, se presentó en el $47 \%$ de los casos.

Cabe resaltar que de los eventos analizados, el 30\% se presentó de forma intrahospitalaria.

\section{Tomografía cerebral inicial y severidad}

El hallazgo más frecuente en la tomografía cerebral, al llegar a urgencias, fue el hematoma intraparenquimatoso $(83,6 \%)$ seguido de la hemorragia subaracnoidea $(9,1 \%)$. De los sangrados intracerebrales cerca del $34 \%$ de los casos presentó drenaje al sistema ventricular y solo el $16 \%$ de los casos una dilatación ventricular asociada al evento hemorrágico.

Fueron más frecuentes aquellos sangrados con localización frontal y hemisférica, además de que el lado izquierdo estuvo comprometido en el $47 \%$ de los casos. La desviación de la línea media, medida como el desplazamiento del septum interventricular con respecto a la línea media de dicho corte axial, fue de más de $5 \mathrm{~mm}$ en cerca del $25 \%$ de los pacientes.

El tamaño del hematoma promedio fue aproximadamente de 39 cc con valores mínimos de 5 cc y máximos de 170 cc.

EI ICH Score, que se utiliza como escala de severidad teniendo en cuenta los hallazgos clínicos y tomográficos, se presentó en cerca del $62 \%$ de los pacientes entre 0 y 1.

\section{Etiología}

La principal causa de los eventos hemorrágicos intracerebrales fueron los trastornos hematológicos en cerca del $40 \%$ de los casos, entre los cuales se cuentan las leucemias, las hemofilias y otros trastornos hematológicos como la aplasia medular, las púrpuras, anemia de Fanconi, entre otros. Como segunda causa del evento, se encuentran las malformaciones arteriovenosas (MAVs) en cerca del $12,7 \%$ de los casos y le siguen las causas neoplásicas en $9 \%$. Dentro de estas últimas causas se encontraron los sarcomas, los gliomas de tallo cerebral y metástasis de tumores germinales. Los aneurismas cerebrales se presentaron en $3(5,5 \%)$ pacientes al igual que las causas autoinmunes, como el lupus y las vasculopatías; y sólo se documentó 1 paciente con una fístula arteriovenosa (FAV) $(1,8 \%)$.

No se identificó una etiología clara en cerca del $25,5 \%$ de los casos, a pesar de estudios complementarios (Figura 1).

\section{Manejo Intrahospitalario}

Teniendo en cuenta los hallazgos clínicos e imagenológi- 


\section{Artículo Original}

\begin{tabular}{|c|c|c|c|}
\hline \multicolumn{4}{|c|}{ Tabla 1. Características base de la población } \\
\hline \multicolumn{2}{|c|}{ Características base de la población $(n=55)$} & \multicolumn{2}{|c|}{ Características base de la población } \\
\hline Edad (media-IQR) & 9,2 años $(5-13) \mathrm{K}-\mathrm{Sp}{ }^{*} 0,006$ & Rigidez nucal & $11 \%$ \\
\hline Género (masculino) & $60 \%$ & Discrasias sanguineas & $47,3 \%$ \\
\hline Escolaridad & & Síntomas al ingreso & \\
\hline Preescolar & $18,2 \%$ & Cefalea/emesis & $36,4 \%$ \\
\hline Primaria & $18,2 \%$ & Deterioro consciencia & $38,2 \%$ \\
\hline Bachillerato & $52,7 \%$ & Convusliones & $9,1 \%$ \\
\hline Ninguno & $10,9 \%$ & Focalización & $5,5 \%$ \\
\hline Año & & Combinación & $10,9 \%$ \\
\hline 2012 & $20 \%$ & Lateralidad & \\
\hline 2013 & $12,7 \%$ & Izquierdo & $47,3 \%$ \\
\hline 2014 & $20 \%$ & Derecho & $25,5 \%$ \\
\hline 2015 & $14,5 \%$ & No aplica & $27,3 \%$ \\
\hline 2016 & $14,5 \%$ & ICH score (moda) & 1 (20 casos) \\
\hline 2017-I & $18,2 \%$ & Vol hematoma (ml)(media-IQR) & $39 \mathrm{ml}(18-55)$ \\
\hline Evento intrahospitalario & $29 \%$ & Manejo QX & $38,2 \%$ \\
\hline GCS (moda) & 15 (25 casos) & Rankin modif (mRs) & \\
\hline Estabilidad hemodinámica & $60 \%$ & $0-2$ & $49,1 \%$ \\
\hline \multirow[t]{2}{*}{ Examen pupilar (isocoria) } & $87,3 \%$ & $3-5$ & $12,8 \%$ \\
\hline & & 6 & $38,2 \%$ \\
\hline
\end{tabular}

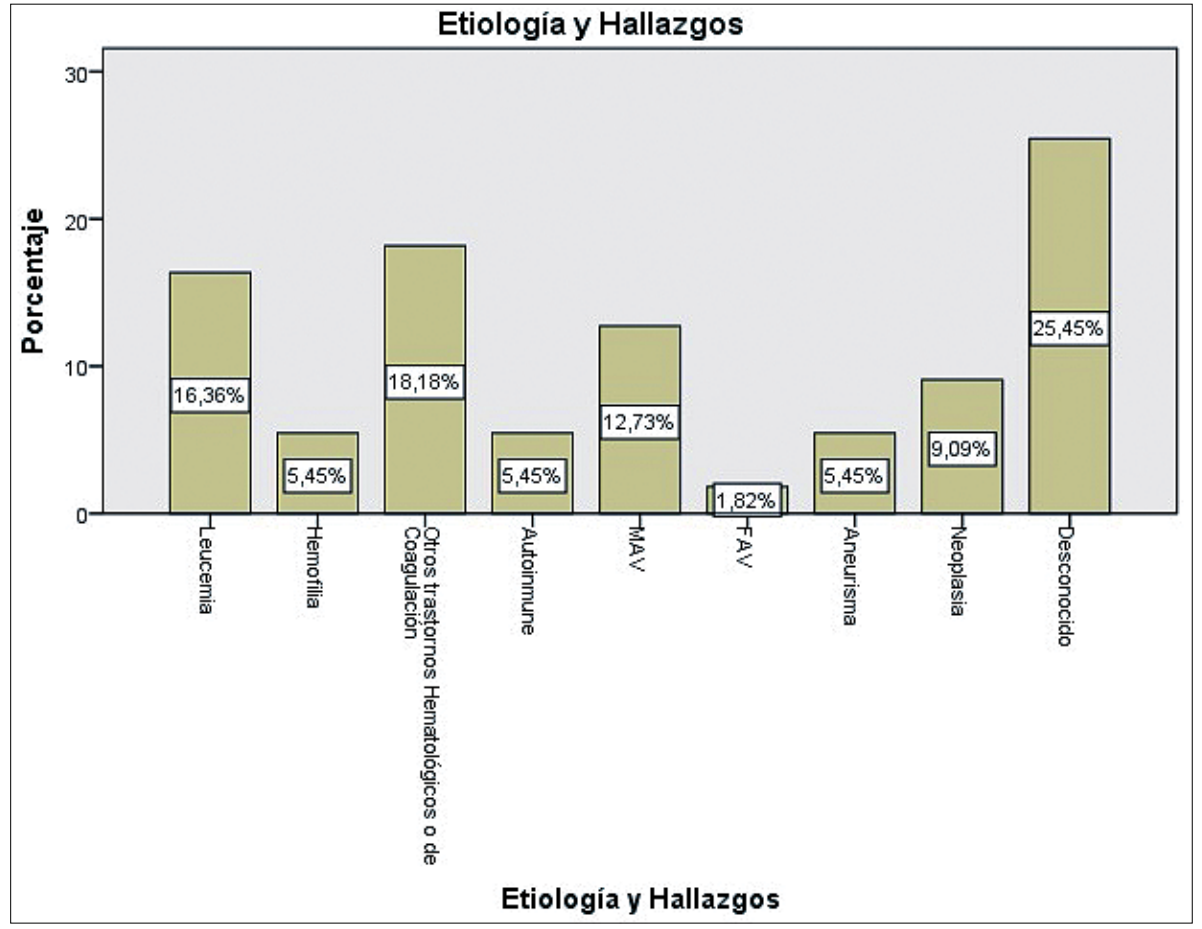

Figura 1. Distribución de los casos por hallazgos etiológicos. cos, el $38 \%$ de los pacientes se llevó a manejo quirúrgico, de los cuales, el $67 \%$ fue de forma urgente, siendo el drenaje del hematoma (sin craniectomía descompresiva) el procedimiento más comúnmente efectuado (69\%).

El drenaje ventricular externo se realizó en el $28,6 \%$ de los pacientes que requirieron manejo quirúrgico. 
En cuanto al manejo complementario, el tratamiento médico de la patología de base fue el más frecuente (69\%) teniendo en cuenta que la primera causa de los eventos hemorrágicos era de carácter hematológico. La resección de las MAVs fue el siguiente procedimiento quirúrgico realizado, en $11 \%$ de los pacientes, seguido de la resección tumoral en $5,5 \%$ y la terapia endovascular en el 3,6\% de los casos.

\section{Discapacidad y mortalidad}

Se utilizó la escala de Rankin Modificada (mRs) para evaluar el estado de discapacidad de los pacientes. El 36,4\% de los casos presentaron una adecuada función posterior al evento ( $m R s$ y 0 ). Rankin de 2 en el $12,7 \%$ de los casos. $\mathrm{Y}$ un compromiso importante de la funcionalidad (mRs 3 a 5) en el $12,8 \%$. Desafortunadamente, el $38,2 \%$ de los pacientes fallecieron (mRs 6).

\section{Correlaciones de Variables}

Teniendo en cuenta los hallazgos, se intentó identificar variables que pudieran estar relacionadas y que pudieran permitir identificar algunos factores que modificaran los resultados y pronósticos de los pacientes estudiados.

Se encontró que existía una relación significativa entre la edad y la mortalidad de los pacientes (Spearman rho $=0,350$ $p=0,009$ ), así como la correlación entre el volumen del hematoma (en $\mathrm{ml}$ ) medido en tomografía y la escala de Rankin modificada para pronóstico (Pearson $r=0,366 p=0,011$ ), el estado neurológico al ingreso y la escala de Rankin modificado (Pearson $r=-0,422 p=0,001$ ) y por último, dicho volumen y el estado neurológico al ingreso a urgencias (Pearson $r=$ $-0,407 p=0,004)$ (Figuras 2, 3 y 4).

\section{Discusión}

El ACV hemorrágico es mucho más frecuente en la población pediátrica comparado con el ACV isquémico, siendo este último más frecuente en los adultos. Se ha evidenciado que el ACV hemorrágico ha alcanzando hasta $45 \%$ de los casos en

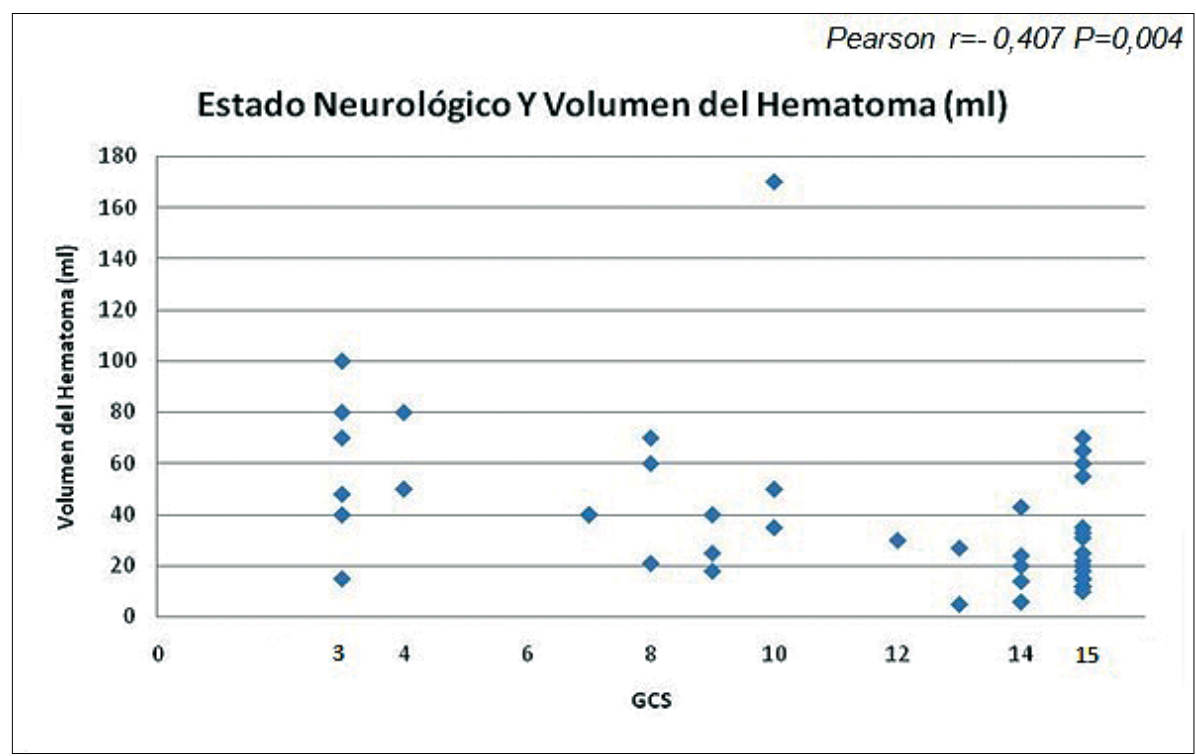

Pearson $r=-0,422 P=0,001$

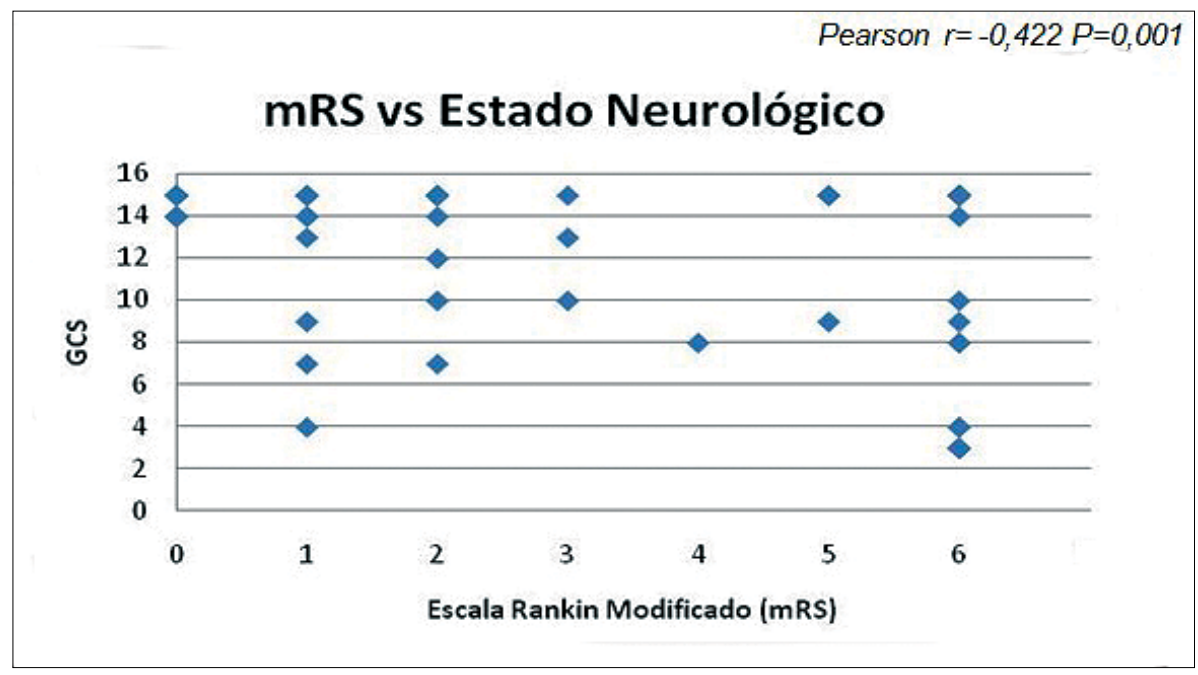

Figura 2. Distribución de los pacientes según estado neurológico en relación con el volumen del hematoma.
Figura 3. Distribución de los pacientes según Escala de Rankin Modificado (mRS) y Estado neurológico. 


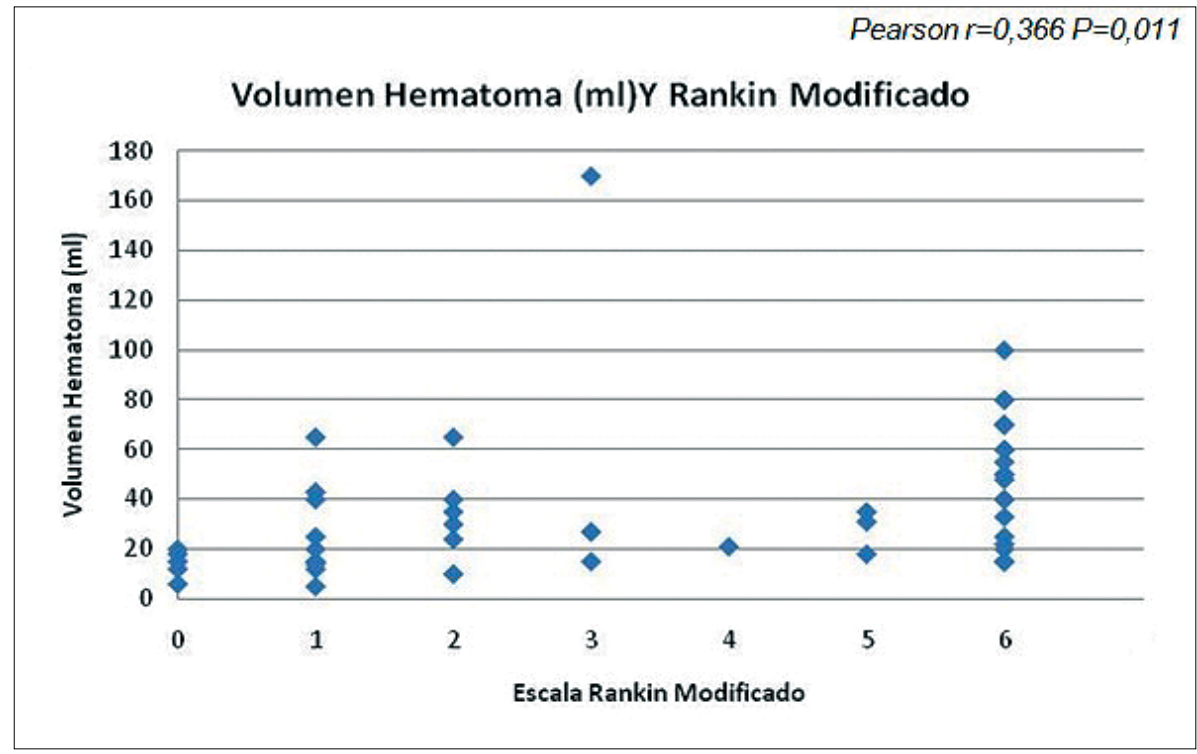

Figura 4. Distribución de los pacientes según Escala de Rankin Modificado (mRS) y volumen del hematoma en $\mathrm{ml}$. pediatría comparado con $10-20 \%$ en los adultos ${ }^{1,2,12}$. Su incidencia estimada, en niños, varía de 1,1 hasta 5,2 por 100.000 mientras que para HSA es de 0,4 por cada $100.000^{1,13}$.

Basados en los datos de algunas bases analizadas, el ACV hemorrágico en niños es una patología que ha sido subestimada pero que compromete cerca del 33 al $54 \%$ de los casos de stroke ${ }^{5}$. De estos, el hematoma intraparenquimatoso y la hemorragia subaracnoidea son los de mayor presentación. Al comparar esta presentación con los adultos, las causas hemorrágicas constituyen del 6,5 al $13 \%$ de todos los strokes ${ }^{1,5,14}$.

La presentación clínica de esta patología no es específica $^{7,9,15}$. Aunque muchos casos pueden presentarse con focalización neurológica o crisis convulsivas como una forma abrupta y llamativa de una patología no especificada del SNC. Por otra parte, la cefalea asociada, o no, a emesis, es una forma de presentación que se asocia con la edad de los pacientes, es decir, algunos estudios evidencian que pacientes con edades menores a los 6 años, no pueden expresar completamente el síntoma de la cefalea hasta que se desarrolla emesis o se compromete la función neurológica1.

Se han descrito varios factores de riesgo y posibles etiologías de los eventos hemorrágicos, como los trastornos hematológicos, principalmente la hemofilia, en pacientes de menor edad, las leucemias, la trombocitopenia y otras etiologías como la anemia de células falciformes, entre otros ${ }^{2,16,17}$.

Las malformaciones arteriovenosas (MAV) tienen una importante participación, llegando, en algunos estudios, a hacer parte de hasta el $47 \%$ de los casos ${ }^{2}$. Se localizan en la región supratentorial (80\%), en la fosa posterior (10\%) y en la línea media $(5-10 \%)^{2,18}$. Pueden ser múltiples en hasta el $17 \%$ de los casos y la probabilidad de sangrado aumenta 2 a $4 \%$ cada año y el riesgo de recurrencia es de $25 \%$ a cinco años. Cabe destacar que un porcentaje menor (8-20\%) presentan síntomas por debajo de los 15 años $^{2}$. En nuestro estudio las MAVs representaron alrededor del $14,5 \%$ incluyendo malformaciones propiamente dichas $(12,7 \%)$ y fístulas durales $(1,8 \%)$.

Los aneurismas intracraneanos son las anormalidades vasculares del sistema nervioso central más comunes, pero su incidencia es menor en niños que en adultos. La prevalencia se estima de 0,5 a $5 \% \%^{1,2,7}$. Son causa importante de hemorragia en adolescentes. Sólo 1-2\% son sintomáticos y están asociados en su mayoría a lesiones vasculares. Su ruptura ocasiona HSA y en algunos casos intraparenquimatosa. Teniendo en cuenta nuestros resultados, los ACVs hemorrágicos de origen aneurismático comprendieron el 5,4\%. Dentro de las características que las diferencian con los adultos son: mayor tamaño, mayor incidencia de aneurismas gigantes y la predominancia es masculina. Dentro de las manifestaciones clínicas se encuentran hemiplejías agudas, signos meníngeos, cefalea severa, náusea, vómito ${ }^{7,9,15}$.

Existe una fuerte relación entre las neoplasias, ya sean propias del SNC o sistémicas, y los ACVs tanto hemorrágicos como isquémicos. Los estudios de Capem et al., demostraron el aumento de 100 veces el riesgo de presentar ACV en pacientes con cáncer comparado con la población general ${ }^{19}$. Sin embargo, las prevalencias de ACV isquémico están alrededor del 1 al $3 \%$ en las series reportadas ${ }^{19}$. Según Kyrnetskiy et al., 30 casos (3\%) de 1.036 pacientes con tumores cerebrales presentaron un ACV hemorrágico mientras que, $18(1 \%)$ de 1.597 pacientes con leucemia presentaron el evento también ${ }^{20}$. La población estudiada en nuestro trabajo, pertenece a un hospital pediátrico de referencia para todo el territorio nacional colombiano, con gran énfasis en patología oncología; en ese orden de ideas, puede existir un aumento de la diferencia entre la etiología del ACV hemorrágico en el contexto neoplásico, al compararlo con los secundarios a MAVs o aneurismas.

La enfermedad autoinmune y, en mucho menor importancia, la hipertensión arterial, se han descrito como otras posibles etiologías en los niños con hemorragia intracerebral ${ }^{21-23}$.

En contraste, y según algunas series reportadas, se desconoce la etiología de los sangrados en hasta el 30 al $50 \%$ de los pacientes ${ }^{1,2,5,7}$. En ese orden de ideas, nuestros hallazgos idiopáticos se encuentren en el rango reportado por la literatura. 
Desafortunadamente, el ACV hemorrágico no tiene un buen pronóstico, siendo la mortalidad alrededor del $25 \%$ de las series reportadas y déficit neurológico posterior entre el 25 y el $57 \%$ de los pacientes ${ }^{1,2,5}$. Nuestro estudio reportó una mortalidad cercana al $38 \%$ y una discapacidad importante de alrededor del $12,8 \%$, medida en la escala de Rankin modificada y teniendo en cuenta los grados de 3 a 5 .

Para la evaluación de la severidad en nuestro estudio se utilizó y extrapoló la escala del ICH score para adultos, teniendo en cuenta su mayor facilidad y reproducibilidad al momento de la toma de los datos. Sin embargo, Beslow et al., propusieron otra posible escala en la que se tienen en cuenta el volumen del hematoma en relación con el volumen cerebral global, generando una puntuación para esa variable. De otra manera, se reemplaza el Glasgow al ingreso y se tienen en cuenta factores como la herniación cerebral y la hidrocefalia ${ }^{24}$. En próximos estudios podría evaluarse la aplicabilidad y reproducibilidad de dicha escala comparada con el ICH score.

En el análisis de datos se evidenciaron variables que pudieron estar relacionadas y que requieren mayores estudios. Independientemente, no se tuvieron en cuenta variables que estaban relacionadas y previamente descritas en la literatura, por ejemplo, el ICH score, relaciona tamaños de hematoma, estado neurológico, localización y mortalidad. Las variables que documentaron una mejor asociación en nuestro estudio fueron la edad y la mortalidad de los pacientes (Spearman rho $=0,350 p=0,009$ ), así como la correlación entre el volumen del hematoma (en $\mathrm{ml}$ ) medido en tomografía y la escala de Rankin modificada para pronóstico (Pearson $r=0,366 p=$ 0,011 ), el estado neurológico al ingreso y la escala de Rankin modificado (Pearson $r=-0,422 p=0,001$ ) y por último, dicho volumen y el estado neurológico al ingreso a urgencias (Pearson $r=-0,407 p=0,004$ ). Estas dos últimas variables fueron reportadas con una importante correlación por Jordan et al., en $2009^{25}$.

Las limitaciones de nuestro estudio incluyeron su naturaleza retrospectiva y la pequeña muestra estadística de pacientes, a pesar de ser un centro exclusivo de atención pediátrica y de que las series más grandes reportadas son similares en el número de casos. Esto debe llevar al desarrollo de nuevos estudios prospectivos y multicéntricos para aumentar el tamaño de la muestra y dar una mayor claridad a asociaciones y factores de riesgo.

\section{Conclusiones}

El ACV hemorrágico es una enfermedad poco estudiada en nuestro país con importantes tasas de complicaciones y mortalidad que afectan a la población pediátrica. Se corroboró el aumento del riesgo de ACV hemorrágico en población pediátrica en pacientes con neoplasias y discrasias sanguíneas, tal como lo corrobora la literatura mundial. Este estudio ha permitido realizar una primera caracterización de dicha problemática con algunas asociaciones de variables, pero se requieren más estudios que permitan realizar mejores correlaciones, seguimientos y pronósticos

\section{Conflictos de interés}

Los autores no tienen ningún tipo de conflictos de interés.

\section{Referencias}

1. Jordan LC, Hillis AE. Hemorrhagic Stroke in Children. Pediatr Neurol. 2007;36:73-80. doi:10.1016/j.pediatrneurol.2006.09.017.

2. Shih E, Beslow L, Editors MS. Hemorrhagic Stroke in Children. Uptodate. 2017:1-22.

3. Lee C, Lin J, Lin K. Clinical Manifestations, Outcomes, and Etiologies of Perinatal Stroke in Taiwan: Comparisons between Ischemic, and Hemorrhagic Stroke Based on 10-year Experience in A Single Institute. Pediatr Neonatol. 2016:1-8.

4. Chantegret $C$, Osseby $G$, Chouchane $M$, et al. Les accidents vasculaires cérébraux du noveau-né et de l' enfant. Stroke in neonates and children. Rev Neurol (Paris). 2009;165:889-900. doi:10.1016/j.neurol.2009.01.039.

5. Lo WD. Childhood Hemorragic Stroke: An Important but Understudied Problem. J Child Neurol. 2013;26(9):1174-1185. doi:10.1177/0883073811408424.Childhood.

6. Lo WD, Lee J, Rusin J, Perkins E, Roach ES. Intracranial Hemorrhage in Children. J Am Med Assoc. 2015;65(12):1629-1633.

7. Meyer-heim AD, Boltshauser E. Spontaneous intracranial haemorrhage in children: aetiology, presentation and outcome. Brain Dev. 2003;25:416-421. doi:10.1016/S03877604(03)00029-9.

8. Zidan I. Intracerebral hemorrhage in children. Alexandria J Med. 2012;48(2):139-145. doi:10.1016/j.ajme.2012.03.001.

9. Al-jarallah A, Al-rifai MT, Riela AR, Roach ES. Nontraumatic Brain Hemorrhage in Children: Etiology and Presentation. J Child Neurol. 1998;15(5):284-289.

10. Srinivasan VM, Gressot L V, Daniels BS, Jones JY, Jea A, Lam S. Management of intracerebral hemorrhage in pediatric neurosurgery. Surg Neurol Int. 2016;7(Suppl 44):S1121-S1126. doi:10.4103/2152-7806.196919.

11. Morgan L. Management of Acute Stroke. Neurol Emergencies. 2007;16(1):5-7.

12. Ahn JH, Wang $\mathrm{K}$, Phi JH, et al. Hemorrhagic moyamoya disease in children: clinical features and surgical outcome. 2012:237-245. doi:10.1007/s00381-011-1535-5

13. Sano K, Ueda Y, Saito I. Subarachnoid Hemorrhage in Children. Childs Brain. 1978;4:38-46.

14. Abbas Q, Merchant A, Nasir B, Haque A, Salam B, Javed G. Spectrum of Intracerebral Hemorrhage in Children: A Report from PICU of a Resource Limited Country. Crit care Res Paractice. 2016;2016:4.

15. Masri A, Al-ammouri I. Clinical presentation, etiology, and outcome of stroke in children: A hospital-based study. 2016;38:204208.

16. Wang J, Shi K, Li J, Jiang L. Risk Factors for Arterial Ischemic and Hemorrhagic Stroke in Childhood. Pediatr Neurol. 2009;40(4):277-281. doi:10.1016/j.pediatrneurol.2008.11.002.

17. Zakhary MM, Wesolowski JR, Sewick AE, et al. Prevalence and Etiology of Intracranial Hemorrhage in Term Children Under the Age of Two Years: A Retrospective Study of Computerized Tomographic Imaging and Clinical. Acad Radiol. 2009;16(5):572577. doi:10.1016/j.acra.2009.01.007.

18. Wanke I, Panagiotopoulos V, Forsting M. Das Risiko intrazerebraler Gefäßmissbildungen. Fortschr Rçntgenstr. 2007;179:365372. doi:10.1055/s-2007-962938.

19. Noje C, Cohen K, Jordan LC. Pediatric Neurology Hemorrhagic and Ischemic Stroke in Children With Cancer. Pediatr Neurol. 
2013;49:237-242.

20. Kyrnetskiy E, Kun L, Boop F, Sanford R, Khan R. Types, causes, and outcome of intracranial hemorrhage in children with cancer. J Neurosurg Pediatr. 2005;102(January 1985):31-35.

21. Zöller B, Li X, Sundquist J, Sundquist K. Risk of subsequent ischemic and hemorrhagic stroke in patients hospitalized for immune- mediated diseases : a nationwide follow-up study from Sweden. BMC Neurol. 2012;12(41):9.

22. Beslow LA, Jordan LC. Pediatric stroke: the importance of cerebral arteriopathy and vascular malformations. Child's Nerv Syst.
2010;26:1263-1273. doi:10.1007/s00381-010-1208-9.

23. Buchanan GR, Adix L. Grading of hemorrhage in children with idiopathic thrombocytopenic purpura. J Pediatr. 2002;141(5):683688. doi:10.1067/mpd.2002.128547.

24. Beslow LA, Ichord RN, Gindville MC, et al. A Simple Grading Scale for Intracerebral Hemorrhage in Children. Stroke. 2014:6671. doi:10.1161/STROKEAHA.113.003448.

25. Jordan LC, Kleinman JT, Hillis AE. Intracerebral Hemorrhage Volume Predicts Poor Neurologic Outcome in Children. Stroke. 2009;40:1666-1671. doi:10.1161/STROKEAHA.108.541383. 